A N N A L E S Annales de Bretagne et des Pays de l'Ouest

\title{
Annie ANTOINE, Julian MischI Sociabilité et politique en
} milieu rural

Rennes, Presses universitaires de Rennes, coll. «Histoire», 472p.

\section{Thibault Marmont}

\section{(2) OpenEdition}

Journals

Édition électronique

URL : https://journals.openedition.org/abpo/236

DOI : $10.4000 / a b p o .236$

ISBN : 978-2-7535-1512-3

ISSN : 2108-6443

Éditeur

Presses universitaires de Rennes

\section{Édition imprimée}

Date de publication : 31 décembre 2008

Pagination : 236-238

ISBN : 978-2-7535-0808-8

ISSN : 0399-0826

\section{Référence électronique}

Thibault Marmont, « Annie AntoIne, Julian Mısch Sociabilité et politique en milieu rural », Annales de Bretagne et des Pays de l'Ouest [En ligne], 115-4 | 2008, mis en ligne le 31 décembre 2009, consulté le 22 juillet 2022. URL : http://journals.openedition.org/abpo/236 ; DOI : https://doi.org/10.4000/abpo. 236

Ce document a été généré automatiquement le 22 juillet 2022.

Tous droits réservés 


\title{
Annie ANTOINE, Julian MischI Sociabilité et politique en milieu rural
}

Rennes, Presses universitaires de Rennes, coll. «Histoire», 472p.

\author{
Thibault Marmont
}

\section{RÉFÉRENCE}

Rennes, Presses universitaires de Rennes, coll. «Histoire», 472p.

\section{NOTE DE L'ÉDITEUR}

Cet ouvrage sur le site des Presses universitaires de Rennes : notice, documents et commande en ligne : http://www.pur-editions.fr/detail.php?idOuv=1788

1 L'ouvrage dirigé par J. Mischi et A. Antoine, qui réunit des textes présentés à un colloque tenu à Rennes en 2005, s'empare d'un double objet de recherche et aspire à extraire les multiples enjeux de ce croisement. Si le pari semblait risqué, du fait du caractère très large, sinon flou, de ces thématiques, il nous semble réussi à plusieurs titres.

2 En plaçant les mondes ruraux et les processus de politisation qui y prennent corps au cœur d'un événement universitaire, les coordonnateurs se sont tout d'abord attachés à réhabiliter un type d'espace (ici moins géographique que social) au sein du débat sociologique et politiste, où il avait progressivement disparu depuis les années 1980. D'autres rencontres de (jeunes) chercheurs cristallisées autour des mêmes problématiques, plus récentes encore ${ }^{1}$, traduisent l'actuel regain d'intérêt de l'ensemble des sciences sociales pour les espaces ruraux. Le colloque «Sociabilité et politique en milieu rural» semble alors constituer un signe précurseur de ce mouvement, qui concourt à endiguer l'urbanocentrisme tendanciel des études politiques contemporaines ${ }^{2}$. 
3 Pour autant, les contributions publiées se démarquent nettement des travaux des sociologues «ruralistes» français et américains des années 1960 et 1970. La finalité affichée est effectivement moins d'enquêter la campagne "pour elle-même ", que de chercher à ré-encastrer des pratiques et des processus politiques ciblés dans leur substrat social et historique. La deuxième qualité essentielle de l'ouvrage réside donc à notre sens dans les éclairages méthodologiques apportés à l'analyse du politique, éclairages dont la portée excède dans une large mesure la catégorie (multiforme) d'espace rural.

4 Ayant vocation à décliner la politisation des ruraux sous l'angle des sociabilités, c'est-àdire in fine au prisme des "transformations des conditions de vie et de travail [des groupes sociaux] et, par conséquent, de leur environnement» (p. 10), les articles sélectionnés adoptent des objets et des focales d'analyse très divers, tant du point de vue des groupes considérés que des terrains étudiés et de la périodicité de l'enquête. Les cinq chapitres qui articulent le recueil traitent successivement de l'appropriation et de la contestation de l'ordre politique, des formes institutionnelles de mobilisation collective, des élites locales et de la compétition électorale, des pratiques d'entraide et des formes de solidarité, et des formes émergentes de sociabilité en milieu rural. Loin de réduire les mondes sociaux des campagnes à la seule sphère paysanne ou agricole, le politique est donc abordé au travers des fêtes publiques, du vote, des notables locaux, de la religion, des politiques d'assistance sociale, des partis, des syndicats, de l'administration... bref, au travers des multiples « ramifications sociales » de la sphère politique institutionnelle (p. 12).

5 Cette pluralité des angles d'approche des processus de politisation est notamment permise par le choix d'une échelle temporelle étendue. Si la moitié des auteurs se sont intéressés aux phénomènes sociopolitiques $\mathrm{du} \mathrm{xx}^{\mathrm{e}}$ siècle, de nombreuses communications portant sur le XIXe siècle, et remontant parfois à l'Ancien Régime et/ou à la Révolution de 1789, apportent au lecteur la preuve que «la politisation des populations rurales renvoie à des processus sociaux longs, même si sa signification peut apparaître de façon plus évidente aux chercheurs lors de conjonctures singulières " (p.19). Enfin, bien que la région bretonne bénéficie d'une attention particulière (un quart des textes lui étant consacrés), on apprécie la perspective comparative qu'introduisent, au plan national, les études issues de terrains allant du Nord-Pas-deCalais au Midi provençal, en passant par la Bourgogne, le Poitou-Charentes, et au plan international, les contributions portant sur le Portugal, l'Espagne, l'Italie, et le SaintEmpire germanique.

6 Ces caractéristiques méthodologiques répondent à un souci épistémologique qui, s'il ne constitue pas à proprement parler l'objet de l'ouvrage, se situe pourtant au principe de sa construction. Le parti pris des coordonnateurs du colloque (respectivement historienne et sociologue) pour la pluridisciplinarité s'inscrit en effet dans la perspective louable d'une « unification des sciences sociales» (p. 7), qu'ont notamment relayé F. Buton, N. Mariot et $\mathrm{G}$. Noiriel au travers du paradigme sociohistorique ${ }^{3}$. Plusieurs articles édités s'ancrent ainsi dans une double filiation, fondée d'une part sur l'analyse archivistique propre à la démarche historienne et, d'autre part, sur une approche compréhensive et constructiviste empruntée à la sociologie. En plaçant le lien social au cœur de l'investigation scientifique du politique dans les espaces ruraux, les chercheurs rassemblés ont donc montré la nécessité d'appréhender conjointement les cadres de l'interaction et l'historicité d'un phénomène pour pouvoir le comprendre. 
7 Devant la complexité et l'ampleur de cette tâche, on ne s'étonnera donc pas que ces actes se referment sur une impression d'inachevé, façonnée en particulier par l'absence regrettable d'une conclusion. La problématique centrale de l'œuvre, consistant à se demander si « la scène politique rurale [peut être], plus qu'en ville, insérée dans des réseaux sociaux infrapolitiques ", n'obtiendra ainsi de réponse (même partielle) qu'au prix de nouveaux travaux de recherche et de nouvelles rencontres scientifiques, dont l'intérêt et la richesse ont été démontrés par les contributeurs de cet ouvrage.

\section{NOTES}

1. Conçu dans le prolongement de cet événement, un colloque international a été organisé en 2006 à Dijon sur la sociabilité et les espaces ruraux : cf. C. Bessière et al., Les mondes ruraux à l'épreuve des sciences sociales, Versailles, éd. Quae, coll. "Symposcience», 2007, 512 pages. Dernièrement, deux rencontres scientifiques ont été consacrées à la spécificité des phénomènes politiques en milieu rural : cf. la journée d'étude "Élu rural: un métier politique comme les autres?», SPIRIT, Bordeaux, 7 avril 2008 (non paru); et le colloque «Battre campagne. Les élections municipales en milieu rural», CEPEL, Montpellier, 11 avril 2008 (à paraître chez L'Harmattan, coll. « Logiques politiques »).

2. Il faut toutefois reconnaître que la discipline historique, dont les recherches sur les mondes ruraux conservent aujourd'hui encore le dynamisme impulsé par M. Agulhon, reste exempte de ce défaut.

3. Voir F. Buton et N. Mariot, «Socio-histoire », Dictionnaire des idées, volume 2 de la collection des « Notionnaires » de l'Encyclopaedia Universalis, 2006, p. 731-733. 